Die angegebenen 4 Farbstoffe können lt. Beschluß der Farbstoff-Kommission der DFG vom 16. und 17. Oktober 1961 zum Färben von Lebensmitteln als annehmbar angesehen werden. Sie entsprechen den allgemeinen Reinheitsanforderungen der Mitteilıng 11 [vgl. diese Z. 123, 124 (1963)]. Es wird besonders darauf hingewiesen, daB die Farbstoffe in Lebensmitteln oder bei der Isolierung aus diesen Veränderungen erleiden können.

Die Ergänzung zu Mitteilung 8 wurde von Dr. H. RAAB (Farbenfabriken Bayer) im Auftrag der Farbstoff-Kommission (Vorsitzender: Professor H. DRUCKREY) zusammengestellt. Sie ist. erhältlich bei der Deutschen Forschungsgemeinschaft (Bad Godesberg, Kennedyallee 40).

S. W. Souci (München)

\title{
Kurznachrichten
}

\section{Kommission für Frnährungsforschung der Deutschen Forschungsgemeinschaft (DFG)}

Über die Gründung der Kommission für Ernährungsforschung der Deutschen Forschungs* gemeinschaft am 10. II. 1958 wurde in dieser Z. 108, I98 (1958) berichtet.

Der Senat der Deutschen Forschungsgemeinschaft hat nunmehr eine Neubesetzung dieser Kommission beschlossen. Der Kommission gehören derzeit folgende Mitglieder an:

Name:

Prof. F. KLose (Kiel), Vorsitzender

\section{Fachgebiet:}

Hygiene

\section{Erzeugung der Nahrung:}

Prof. G. Aufhammer (Weihenstephan)

Prof. E. Mückenhausen (Bonn)

Prof. W. Lenkert (Göttingen)

Prof. H. RICHTER (Braunschweig)
Acker- und Pflanzenbau

Bodenkunde

Tierernährung

Pflanzenschutz

\section{Medizinische Fragen:}

Prof. H. D. Cremer (Gießen)

Prof. H. Holzer (Freiburg i. Br.)

Prof. H. KraUt (Dortmund)

Prof. J. KüHNAU (Hamburg)

Prof. M. Lerche (Berlin)

Prof. J. Jоснтмs (Lübeck)

Prof. K. OBERDISSE (Düsseldorf)

Prof. N. ZöLLiner (München)
Ernährungsphysiologie

Physiologische Chemie

Physiologische Chemie

Physiologische Chemie

Mikrobiologie (vet.)

Pädiatrie

Innere Medizin

Innere Medizin

3. Lebensmittelchemie und -technologie:

Prof. K. G. Bergner (Stuttgart)

Prof. W. DieMraIR (Frankfurt a. M.)

Prof. J. SCHORMÜLLER (Berlin)

Prof. S. W. Souci (München)
Lebensmittelchemie

Lebensmittelchemie

Lebensmittelchemie und -technologie

Lebensmittelchemie

$\mathrm{Zu}$ den Sitzungen der Kommission werden im Bedarfsfall die Leiter der Untergruppen im Schwerpunkt ,Ernährungsforschung ${ }^{6}$ eingeladen. Es bestehen zur Zeit folgende Untergruppen:

\section{Name der Leiter:}

Prof. H. OPITz (Heidelberg)

Prof. H. GLatzes (Dortmund)

Prof. J. BrügGEMANN (München)

Prof. B. Rademacher (Stuttgart)

Prof. H. REPLOH (Münster i. W.)

Prof. J. SchormülLer (Berlin)
Untergruppe:

Kinderernährung

Ernährung des älteren Menschen

Stoffe mit Sonderwirkung in ihrer

Bedeutungfür Mensch und Tier

Pflanzenschutzmittel

Mikrobiologie

Lebensmittelvorratshaltung nach

Bedarf, Auswahl und Lagerung. S. W. Souci (München) 